\title{
Annealing and temperature coefficient study of type IA fibre Bragg gratings inscribed under strain and no strain - implications to optical fibre component reliability
}

\author{
Kyriacos Kalli ${ }^{1 *}$, George Simpson ${ }^{2}$, Helen Dobb ${ }^{2}$, Michael Komodromos ${ }^{3}$, David Webb ${ }^{2}$ and \\ Ian Bennion ${ }^{2}$ \\ ${ }^{1}$ Higher Technical Institute, C. Kavafi Street, Nicosia, 2152 Cyprus \\ ${ }^{2}$ Photonics Research Group, Aston University, Birmingham B4 7ET United Kingdom \\ ${ }^{3}$ Frederick Research Centre, 7 Filokyprou, Nicosia, Cyprus
}

\begin{abstract}
The annealing properties of Type IA Bragg gratings are investigated and compared with Type I and Type IIA Bragg gratings. The transmission properties (mean and modulated wavelength components) of gratings held at predetermined temperatures are recorded from which decay characteristics are inferred. Our data show critical results concerning the high temperature stability of Type IA gratings, as they undergo a drastic initial decay at $100^{\circ} \mathrm{C}$, with a consequent mean index change that is severely reduced at this temperature However, the modulated index change of IA gratings remains stable at lower annealing temperatures of $80^{\circ} \mathrm{C}$, and the mean index change decays at a comparable rate to Type I gratings at $80^{\circ} \mathrm{C}$. Extending this work to include the thermal decay of Type IA gratings inscribed under strain shows that the application of strain quite dramatically transforms the temperature characteristics of the Type IA grating, modifying the temperature coefficient and annealing curves, with the grating showing a remarkable improvement in high temperature stability, leading to a robust grating that can survive temperatures exceeding $180^{\circ} \mathrm{C}$. Under conditions of inscription under strain it is found that the temperature coefficient increases, but is maintained at a value considerably different to the Type I grating. Therefore, the combination of Type I and IA (strained) gratings make it possible to decouple temperature and strain over larger temperature excursions.
\end{abstract}

Keywords: Fibre reliability, fibre Bragg gratings, annealing, Type IA grating, photosensitivity

\section{INTRODUCTION}

Type IA fibre Bragg gratings have attracted interest within the sensor community based on their unique spectral and physical characteristics. They are readily identified by their spectral attribute of a large red shift in the Bragg wavelength $\left(\lambda_{\mathrm{BR}}\right)$ of the grating during inscription that accompanies an increase in the mean core index ${ }^{1}$. It is recognised that this red shift is dependent on fibre type and hydrogenation conditions, and for a highly doped fibre, such as B/Ge codoped fibre, is typically $15-20 \mathrm{~nm}$, whereas the wavelength shift for SMF28 is lower at $5-8 \mathrm{~nm}^{2}$. The maximum wavelength shift translates to an increase in the mean index of up to $2 \times 10^{-2}$. A strong correlation between the growth of the $\mathrm{OH}$ absorption band formation in the optical fibre during prolonged UV exposure and the increase in the mean index change of the fibre grating has been reported, resulting in a fundamental material modification so that Type I and Type IA gratings have been written in the same fibre with a common phase mask, yet with central reflecting peaks more than $14 \mathrm{~nm}$ apart after annealing ${ }^{3}$.

\section{INSCRIPTION PROCEDURE}

Gratings were inscribed using the scanning phase mask technique and the fibre was illuminated with a cw UV Coherent Sabre FreD laser source operating at $244 \mathrm{~nm}$, Figure 1. A $1 \mathrm{~mm}$ aperture ensured an accurate top-hat exposure profile along the length of the grating ${ }^{3}$. The stage was scanned at $1 \mathrm{mms}^{-1}$ with $130 \mathrm{~mW}$ optical power delivered to the fibre.

Reliability of Optical Fiber Components, Devices, Systems, and Networks III, edited by Hans G. Limberger, M. John Matthewson,

Proc. of SPIE Vol. 6193, 61930L, (2006) - 0277-786X/06/\$15 - doi: 10.1117/12.662318

Proc. of SPIE Vol. 6193 61930L-1 


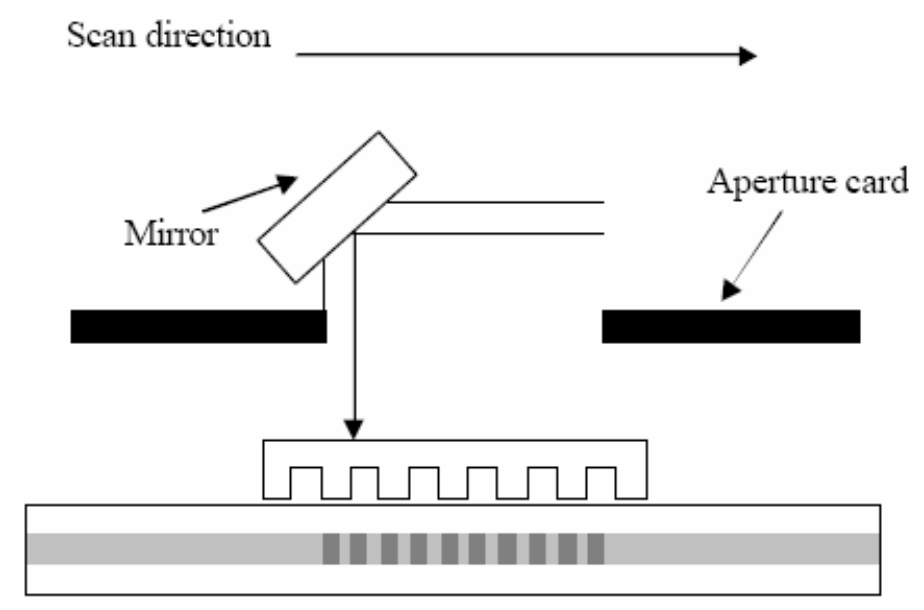

Figure 1. Scanning beam inscription setup using a phase mask and an aperture card to ensure uniform grating exposure.

The grating manufacture was a two-step process; first the phase mask was removed and the UV beam scanned along the fibre length to give a blank beam exposure. The mask was then reintroduced and a grating written into the UV preexposed section of fibre. This has proven the most successful way of inscribing Type IA gratings with a clean wavelength spectrum. The fibre underwent hydrogenation as follows; two identical batches of fibre were prepared and each batch consisted of SMF28 (standard corning fiber), B/Ge co-doped fibre (FibercorePS1250/1500 B/Ge co-doped single mode fibre) and two different samples of Ge-only doped fibre (Verillion $12 \mathrm{~mol} \% \mathrm{Ge}$ and $21 \mathrm{~mol} \% \mathrm{Ge}$ ). One batch was hydrogenated at $80^{\circ} \mathrm{c}, 190 \mathrm{bar}$ for 93 hours and cooled to room temperature over 24 hours by which time the pressure was 160bar; the other batch was hydrogenated in excess of four months at $180 \mathrm{bar}$ and at room temperature. The hydrogen concentration ${ }^{4}$ within the fibre samples based on the hydrogenation conditions outlined above is 11400 and 21000 ppm, respectively.

In order that the IA gratings have a reference grating whose properties are well understood, a $1 \mathrm{~mm}$ Type I grating was written within the same section of each fibre. Figure 2 shows the spectra of each sample before (upper) and after (lower) annealing at $80^{\circ} \mathrm{C}$ for 96 hours, and highlights a number of differences between the fabricated gratings; notable examples are the gratings written in Verillion B/Ge co-doped fibre, where both the IA and I gratings differ significantly in amplitude and the Type I grating would seem to be slightly offset in wavelength. The gratings in SMF28 are comparable in hot and cold hydrogenated samples and do not exhibit any significant spectral characteristics.
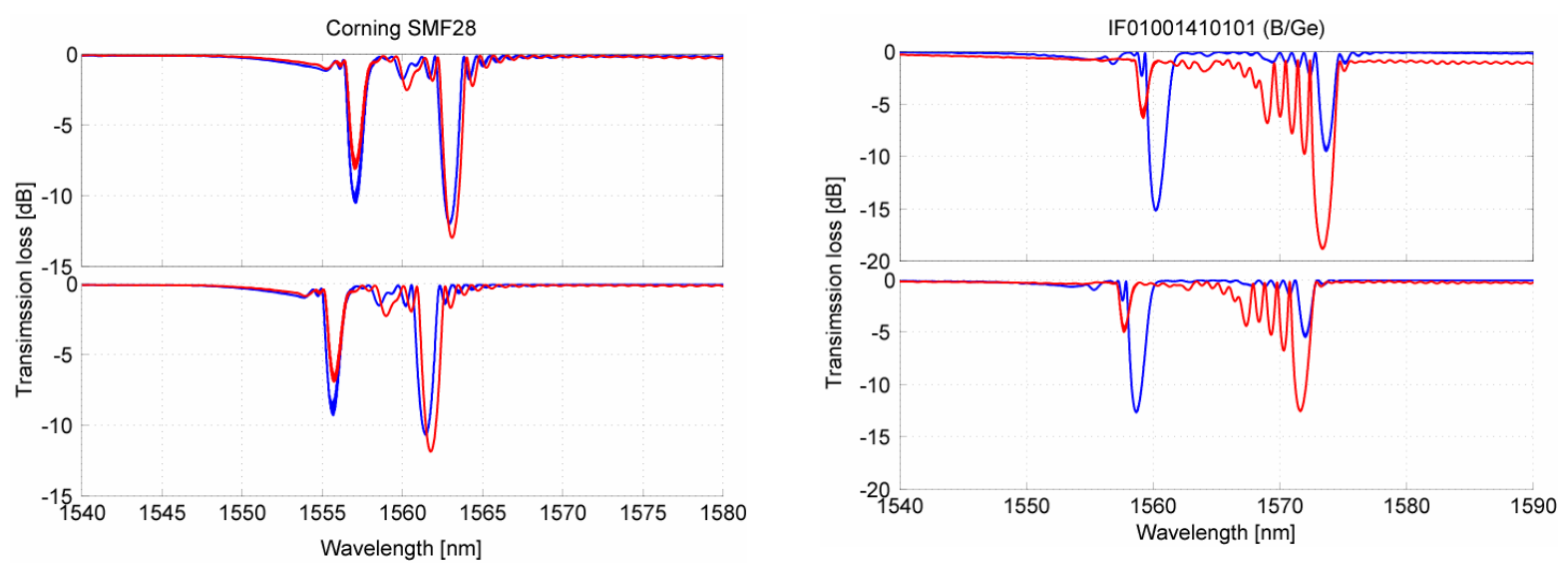

Figure 2. Pre- (upper traces) and post- (lower traces) annealing spectra for Type I and Type IA gratings written in the indicated fibre types for hot (grey) and cold (black) hydrogenation conditions. 


\section{DECAY OF UV INDUCED GERMANOSILICATE GRATINGS}

Williams and Smith ${ }^{5}$ studied the thermal stability of strong and weak gratings. They showed that proper stabilisation by annealing ensures that the gratings remain extremely stable and may be expected to last at least 25 years, this result was confirmed by Kannan et $a l^{6}$ among others. Ishikawa et $\mathrm{l}^{7}$ proposed a model by which the proper annealing time may be calculated. Patrick et al ${ }^{8}$ compared the stability of hydrogenated and non hydrogenated germanosilicate fibres and concluded that gratings written in hydrogen free fibre were significantly more stable at elevated temperatures. Aslund et $a l^{9}$ showed that presensitised fibres are more thermally stable than hydrogenated fibres. Niay et al ${ }^{10}$ showed that UV grating inscription using either $\mathrm{CW}$ or pulsed sources resulted in gratings possessing the same elevated temperature stability properties (for the same grating types, and after proper annealing).

\subsection{Modulated index changes during annealing}

The modulated index change may be represented by the integrated coupling constant (ICC) in preference to reflectivity or peak transmission loss because this quantity is proportional to the UV-induced refractive index change, even for cases where the gratings are non-uniform. This quantity may be calculated according to ${ }^{11}$ :

$$
I C C=\tanh ^{-1} \sqrt{1-T_{\min }}
$$

where $T_{\min }$ is the transmission minimum, i.e. the grating peak reflectance $R=1-T$. It is possible to normalise $I C C$ plots according to grating length,

$$
I C C^{\prime}=\frac{I C C}{L}
$$

or initial amplitude,

$$
I C C^{\prime \prime}(t)=\frac{I C C_{t=0}}{I C C(t)}=\eta
$$

or both which enables the direct comparison of gratings with different lengths or initial reflectance. Erdogan et al ${ }^{11}$ showed that initially gratings decay extremely quickly which is followed by a substantial decrease in the rate of decay at longer times. They concluded that this behaviour was indicative of a power law dependence on time and could be fitted according to ${ }^{11}$ :

$$
\eta=\frac{1}{1+A\left(t / t_{1}\right)^{\alpha}}
$$

where the factor $A$ and exponent $\alpha$ depend on temperature and are dimensionless, whilst $t_{l}=1 \mathrm{~min}$ in order to keep dimensions consistent. Baker et al ${ }^{12}$ confirmed the power law thermal decay model holds well for non-hydrogenated fibres but went on to suggest that for hydrogenated fibres it was a poor fit, relying instead on a log time representation that was indicative of a broader trap distribution with a top hat profile,

$$
\eta=1-K \log \left(\frac{t}{\tau}\right)
$$

where $K$ and $\tau$ are constants and $\tau$ is temperature dependent obeying a classic Arrhenius relationship 


$$
\tau=A e^{E_{a} / R T}
$$

which is characteristic of the aging of glass.

\subsection{Mean index changes during annealing}

A study of the wavelength shift of FBGs manufactured in hydrogenated germanosilicate (Corning SMF28) fibre by Masuda $\mathrm{et} \mathrm{al}^{13}$ showed that the wavelength shift during annealing was solely due to the out-gassing of $\mathrm{H}_{2}$ from the fibre. This was shown by annealing a specimen grating then re-hydrogenating it and then annealing it again. During both annealing processes the wavelength evolution was shown to be the same, and hence they concluded their result. However, Chisholm et al ${ }^{14}$ have showed that for boron germanium co-doped silica fibres, the wavelength decay still occurred after the hydrogen had out-gassed. Pal et al ${ }^{15}$ have showed that the power law model used to model modulated index decay may be modified to model the decay in mean index.

\section{ANNEALING STUDY}

\subsection{Experimental arrangement for monitoring annealing}

The annealing properties of Type IA gratings were studied in order to investigate their thermal stability and any similarities to the annealing properties of other grating types. The transmission profile of several gratings was recorded at predetermined temperatures using an 8 channel DWDM test system comprising a tuneable laser with $5 \mathrm{pm}$ step size, a wavemeter, launch power meter and a rack of $8 \mathrm{InGaAs}$ photodiodes with $>100 \mathrm{~dB}$ dynamic range. The tuneable laser was connected to the wavemeter, before feeding the samples such that a highly accurate measure of the discrete wavelength might be obtained. The samples were placed in a Sanyo environmental chamber $\left(-40^{\circ} \mathrm{C}\right.$ to $180^{\circ} \mathrm{C}$ range) . This apparatus is shown schematically in Figure 3. After running such an experiment the individual traces were processed using another custom software program which calculated the Bragg wavelength and peak transmission loss for each dataset.

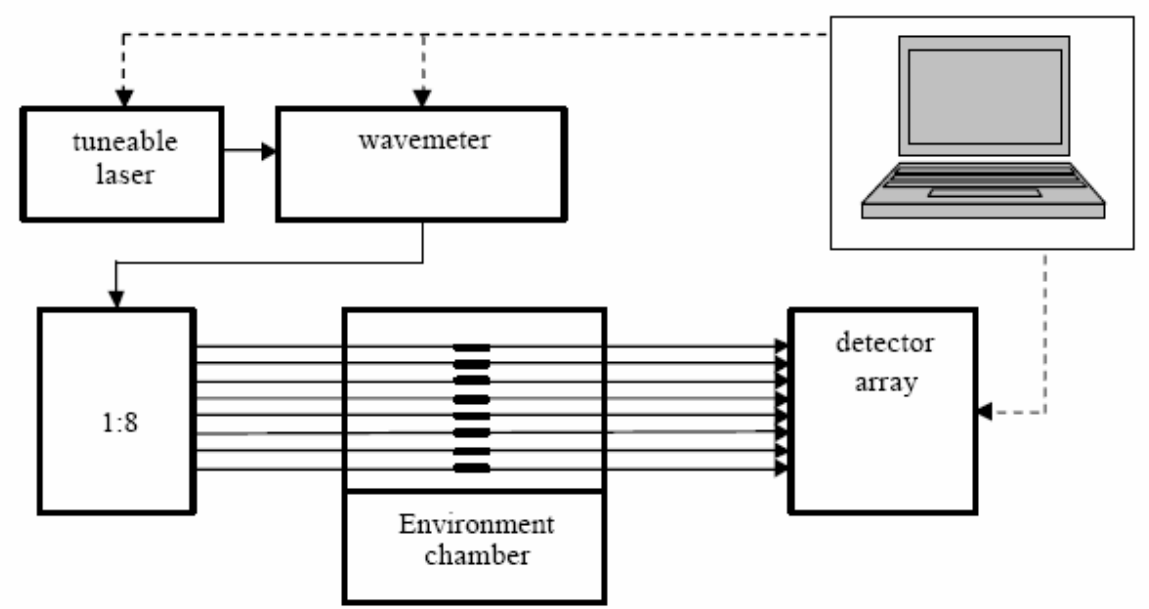

Figure 3. Schematic showing the tuneable laser system used to characterise the gratings during the heating cycle in the tubular oven. This system enabled 5pm resolution with $100 \mathrm{~dB}$ dynamic range on all 8 channels.

\subsection{Standard annealing at $80^{\circ} \mathrm{C}$ of IA-I dual sensors}

This subsection details the annealing data for 8 IA-I dual grating sensors manufactured in $\mathrm{B} / \mathrm{Ge}$ fibre (Verillion IF01001410101), hydrogenated at $200 \mathrm{Bar} / 80^{\circ} \mathrm{C}$ for 64 hours. The Type I grating was $1 \mathrm{~mm}$ long and was written at $0.5 \mathrm{mms}^{-1}$ whereas the Type IA grating was $4 \mathrm{~mm}$ long and written at $0.05 \mathrm{mms}^{-1}$ after 200 blank beam scans at $1 \mathrm{mms}^{-1}$ to induce the mature IA index change. 

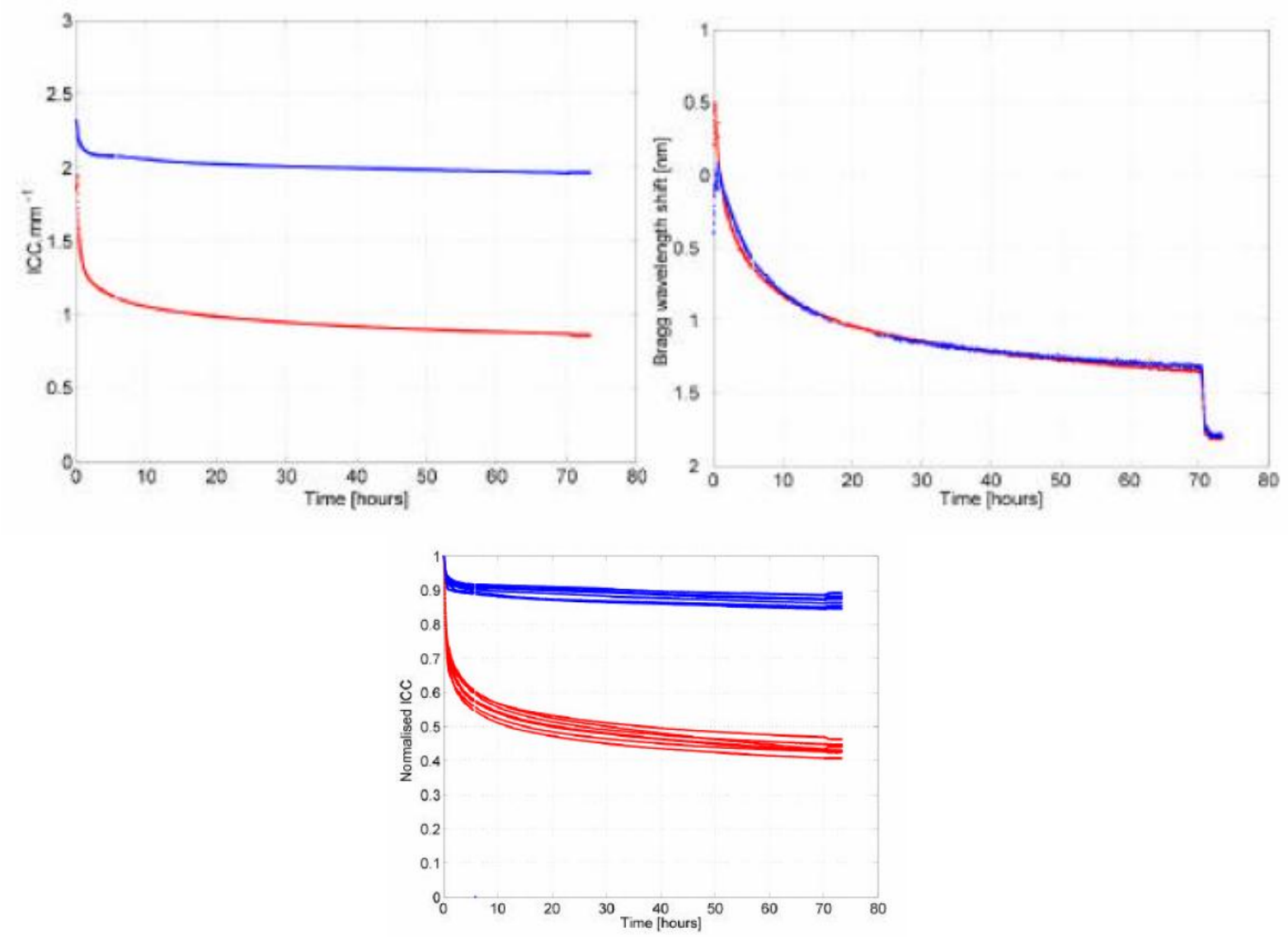

Figure 4. (a) (left-upper) The $80^{\circ} \mathrm{C}$ annealing curves for Type I (black-UPPER) and Type IA (grey-LOWER) gratings for sample reference FBG4 shown in terms of the ICC per unit length. (b) (right-upper) The annealing curves shown in terms of the Bragg wavelength shift during annealing. (c) (bottom-centre) The $80^{\circ} \mathrm{C}$ annealing curves for Type I (black-UPPER) and Type IA (grey-LOWER) gratings for 8 samples shown in terms of the normalised ICC.

The gratings were annealed at $80^{\circ} \mathrm{C}$ for 70 hours. Figure 4 shows the annealing curves for an example grating (FBG7). In Figure 4a the annealing curves for the two gratings in terms of ICC. $\mathrm{mm}^{-1}$. This plot explicitly shows that the Type I grating anneals significantly more slowly than the IA grating. Figure $4 \mathrm{~b}$ shows the decay of the mean index during the annealing process as metered by the Bragg wavelength of the grating during the annealing. This figure shows that in this case, both Type I and IA gratings undergo the same rate of mean index change. The data indicates that their mean indices anneal similarly and this is principally due to hydrogen out-gassing that is set by the conditions of the environment chamber. After 70 hours we observe the reversible wavelength shift that results from the temperature decrease. Figure $4 \mathrm{c}$ confirms that the annealing curves for many samples of both grating types show significant differences between them.

It is well documented that gratings written in hydrogenated fibres have a broader trap distribution and this is manifest from the larger index change observed for gratings in this fiber type. The actual distribution is not apparent, although studies by Rathje et al ${ }^{16}$ point to decay from two different defects. Our study shows that both the Type I and Type IA gratings are characterized by a second order exponential decay but with different time constants. The current annealing data indicates that standard Type I gratings are more robust than their Type IA counterparts at an elevated temperature. In general, amorphous materials display multiple relaxation mechanisms, described by a summation of exponential equations; the decay of the fibre Bragg grating is a special case of this decay process. Fitting to this data indicates an excellent fit with a second order exponential decay, Figure 5. This is in keeping with the broader distribution of trap states for gratings in hydrogen loaded fibre, which extend to low energy states that are subject to faster decay at the annealing temperature. The data was fitted with a function of the form, 


$$
y=y_{0}+A_{1} e^{-x / t_{1}}+A_{2} e^{-x / t_{2}}
$$

which is a standard dual exponential decay function representing relaxation mechanisms with two characteristic decay times, Table 1.
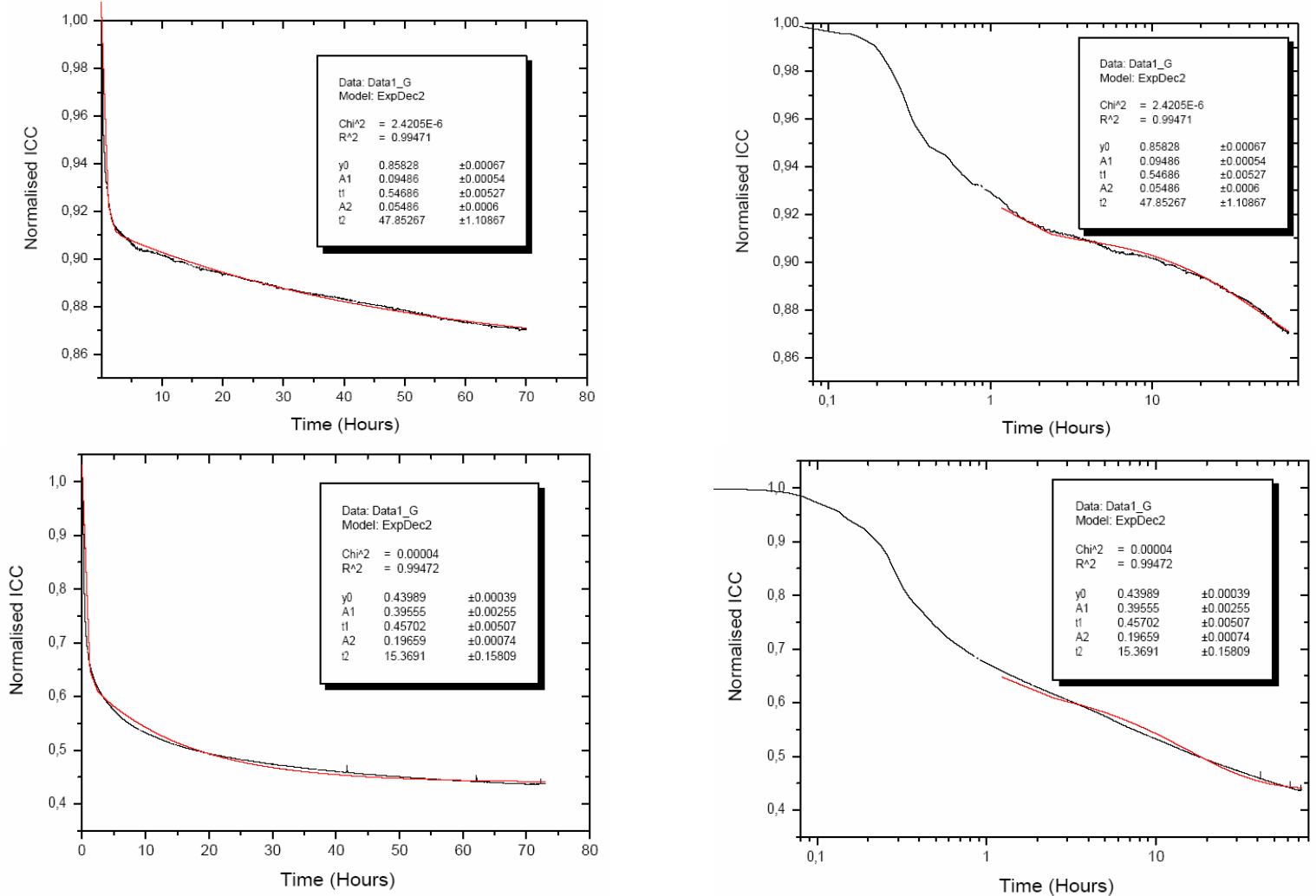

Figure 5. The $80^{\circ} \mathrm{C}$ annealing curves for Type I gratings with a linear time scale (upper left) and log time scale (upper right) and IA gratings with a linear time scale (lower left) and log time scale (lower right) shown with a representative fit to the normalised coupling coefficient with time.

\begin{tabular}{c|c|c}
\hline & Type I Grating & Type IA Grating \\
\hline $1^{\text {st }} \begin{array}{c}\text { decay time } \\
(\mathrm{hr})\end{array}$ & 0.547 & 0.457 \\
$2^{\text {nd }} \begin{array}{c}\text { decay time } \\
(\mathrm{hr})\end{array}$ & 47.85 & 15.37 \\
\hline
\end{tabular}

Table 1 Characteristic decay times for the Type I and IA gratings, based on data fitting using equation (7).

\subsection{High temperature stability}

In order to evaluate the temperature stability of Type IA gratings an experiment was designed to make a direct comparison between Types I, IA and IIA. The test looked at six Type I, six Type IA and two Type IIA gratings. The fibre used in this trial was Fibercore PS1250/1500 B/Ge co-doped single mode fibre (serial number 30246B/A-00A). The Type I and IA gratings were fabricated in fibre hydrogenated at $200 \mathrm{bar}$ at $80^{\circ} \mathrm{C}$ for 64 hours. The IA gratings were manufactured according to the blank beam exposure method detailed earlier; a $3 \mathrm{~mm}$ section of fibre was pre-exposed by $244 \mathrm{~nm}$ radiation at $1 \mathrm{mms}^{-1}$ until the $1400 \mathrm{~nm}$ absorption band had reached a maximum value. A $2 \mathrm{~mm}$ FBG was then written using a scanning phase mask technique at $0.1 \mathrm{mms}^{-1}$. A $2 \mathrm{~mm}$ Type I grating was then inscribed with a $1 \mathrm{~mm}$ 
separation from the IA grating at $2 \mathrm{mms}^{-1}$. The Type IIA gratings were fabricated by the shuttered scanning phase mask method in non-hydrogenated fibre (from the same PS1250/1500 batch) with a scan speed of $1 \mathrm{mms}^{-1}$ and a shutter width of $2 \mathrm{~mm}$. A typical spectrum of each grating is shown in Figure 6 for gratings manufactured to have the same strength but different length, hence the different grating bandwidths. All gratings were manufactured in the middle of a $1 \mathrm{~m}$ section of fibre. The gratings were placed inside a high temperature Carbolite tubular furnace in place of the Sanyo environmental chamber. The gratings were held at $100^{\circ} \mathrm{C}, 200^{\circ} \mathrm{C}, 300^{\circ} \mathrm{C}, 400^{\circ} \mathrm{C}$ and $500^{\circ} \mathrm{C}$, although we present data for the first two temperatures only, as these are pertinent to the results of this study.

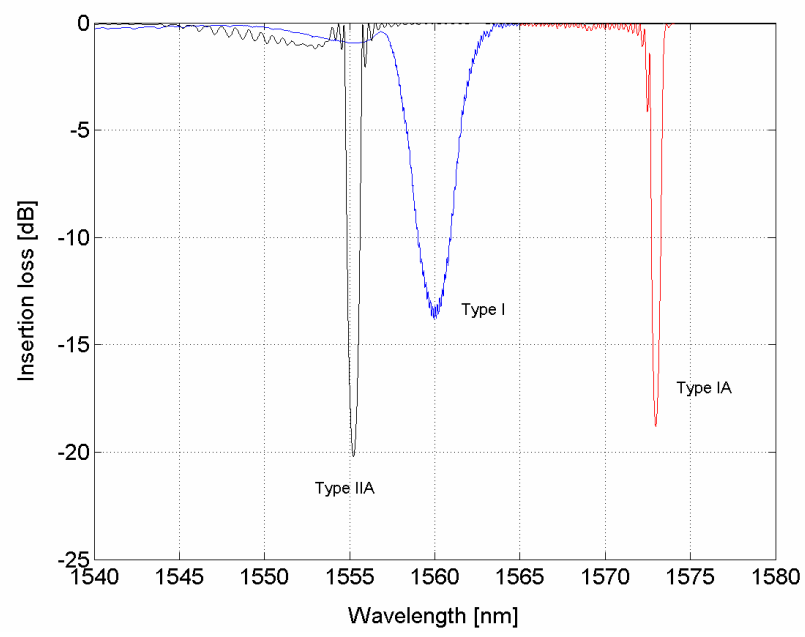

Figure 6. Spectra of Type I, IA, and IIA gratings prior to annealing written in B/Ge fibre. Each grating has the same period, but dissimilar lengths, and demonstrates the differences in the mean index of the three gratings.

In order to ascertain the thermal stability of the gratings the mean and modulated index changes of the samples were calculated. This was done by means of a software program designed to sift through the 8000 or so individual spectral traces and produce tables of the central reflecting wavelengths and peak insertion losses for each grating as a function of time. This information was then converted to mean and modulated index change as follows:

\section{Mean index change}

The mean index change was calculated according to the following rearrangement:

$$
\begin{gathered}
\lambda_{B R}=2 n_{\text {eff }} \Lambda \\
\Delta n_{\text {eff }}(t)=\frac{\lambda_{B R(t)}}{2 \Lambda}-n_{\text {initial }}
\end{gathered}
$$

where $\Delta n_{\text {eff }}$ is the effective refractive index at time $t, \lambda_{B R}$ is the Bragg wavelength at time $t, n_{\text {initial }}$ is the initial refractive index of the fibre core (supplied by the fibre manufacturer as 1.4441) and $\Lambda$ is the grating period. $\Lambda$ may be accurately approximated as follows:

$$
2 \Lambda=\frac{\lambda_{\text {initial }}}{n_{\text {initial }}}
$$

where $\lambda_{\text {initial }}$ is found by inscribing a Type I grating in non-hydrogenated fibre. Care should be taken so that the grating is sufficiently immature that it has not exhibited a significant level of the red shift seen during the earliest stages of grating 
manufacture. This is shown in Figure 7, which yields a value of $1077.515 \mathrm{~nm}$ for twice the period of the gratings when applied to Equation 10.

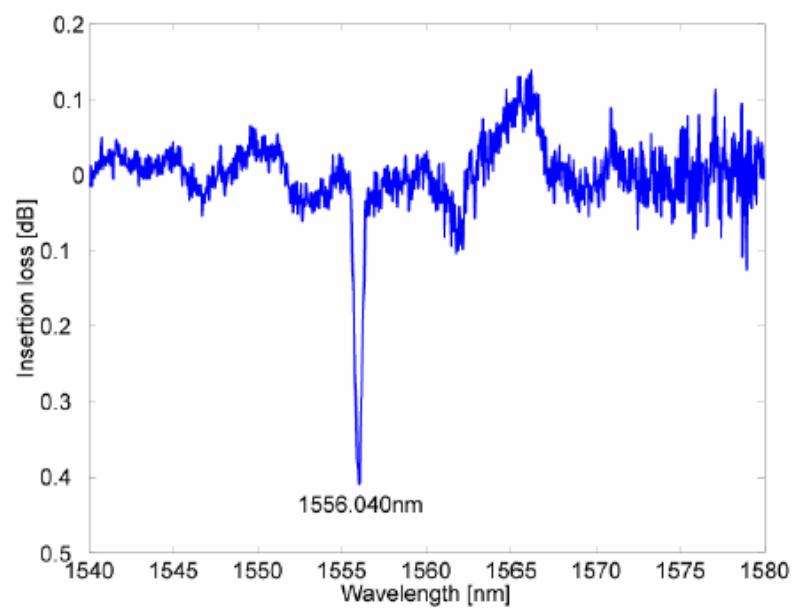

Figure 7. $\lambda_{\mathrm{BR}}$ of a Type I grating in non-hydrogenated Fibercore PS1250/1500 with $\mathrm{n}_{\mathrm{eff}}=1.4441$ as used to calculate the period of the inscribed gratings during the annealing experiment.

It is also desirable to normalise the recorded values of central reflectance for the effects of thermal shift in the Bragg resonance. This is most simply done by applying $\lambda_{B R}(t)=T(t) \lambda_{B R}^{\prime}(T)$, where $T(t)$ is the temperature at time $t$, and $\lambda_{B R}{ }_{B R}(T)$ is the thermal coefficient of the individual gratings. The average values shown in Table 2 were used, since it was not possible to measure the thermal coefficients of these particular samples, as the high final temperatures used in this experiment carbonised the fibre beyond use.

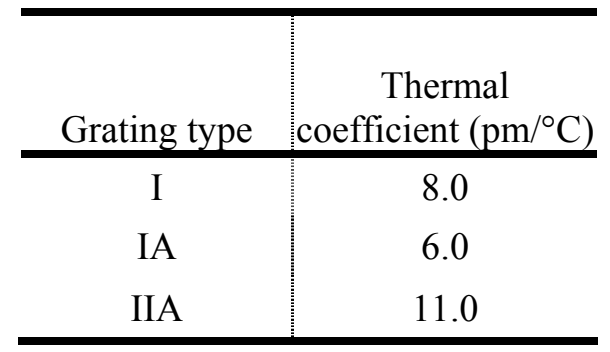

Table 2 Summary of average thermal coefficients used to calculate the shift in the Bragg resonances during annealing. This data represents an approximation used to normalise the mean index change under different temperatures.

The result of Equation 10 was substituted back into Equation 9 and normalised to $t=0$ for successive values of $\lambda_{B R}$ thus producing a dataset containing the mean index change against time for the gratings at increasing ambient temperature. This data is shown in Figure 8 below. 

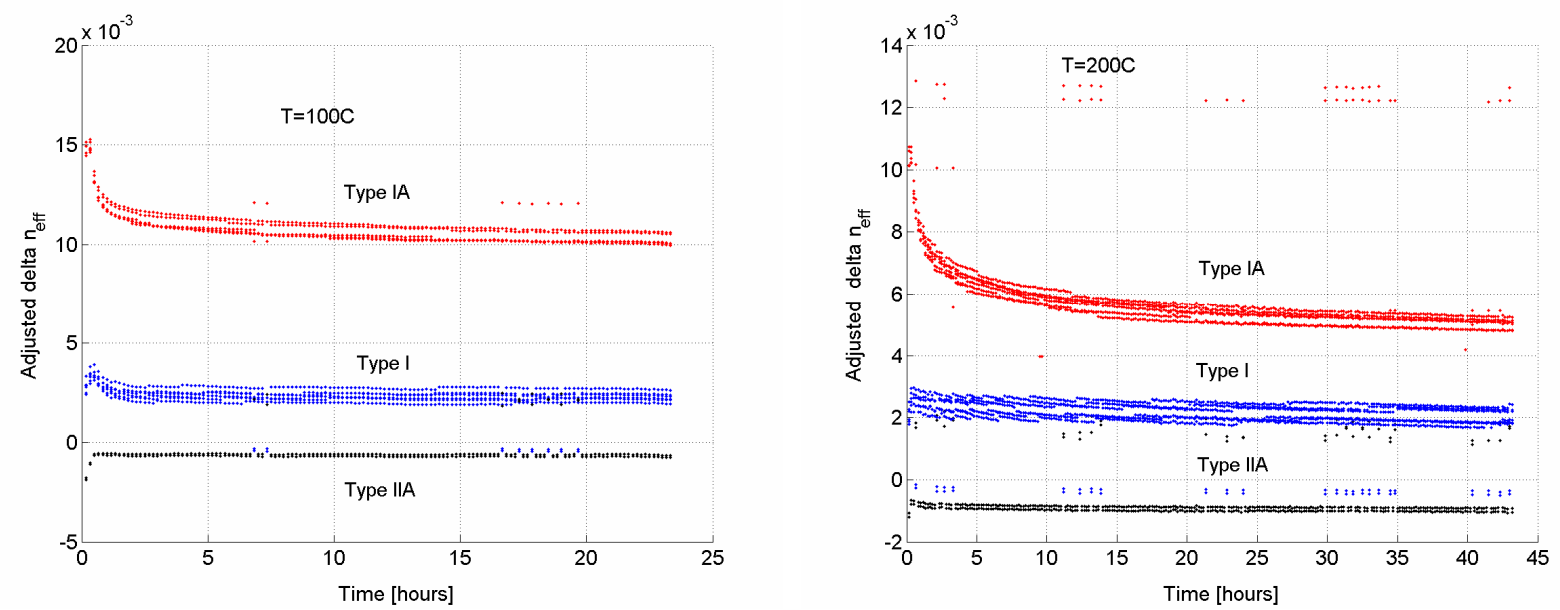

Figure 8. The decay in the mean index experienced by Type IA (top), Type I (middle) and Type IIA (bottom) gratings during heating at $100 \mathrm{C}$ (left), $200 \mathrm{C}$ (right). This figure is the first evidence that the mean index change of IA gratings also decays under moderate temperatures.

Figure 8 shows that the mean index change is severely reduced at a temperature of $100^{\circ} \mathrm{C}$. However, the results also indicate that the modulated index change of IA gratings remains stable at lower annealing temperatures of $80^{\circ} \mathrm{C}$, and the mean index change decays at a comparable rate to Type I gratings at $80^{\circ} \mathrm{C}$, as indicated in Figure 4 . These results show that the maximum temperature IA gratings may be reliably used is less than $80^{\circ} \mathrm{C}$.

\section{Modulated index change}

The modulated index change was shown in terms of the normalised ICC, and is shown below in Figure 9.
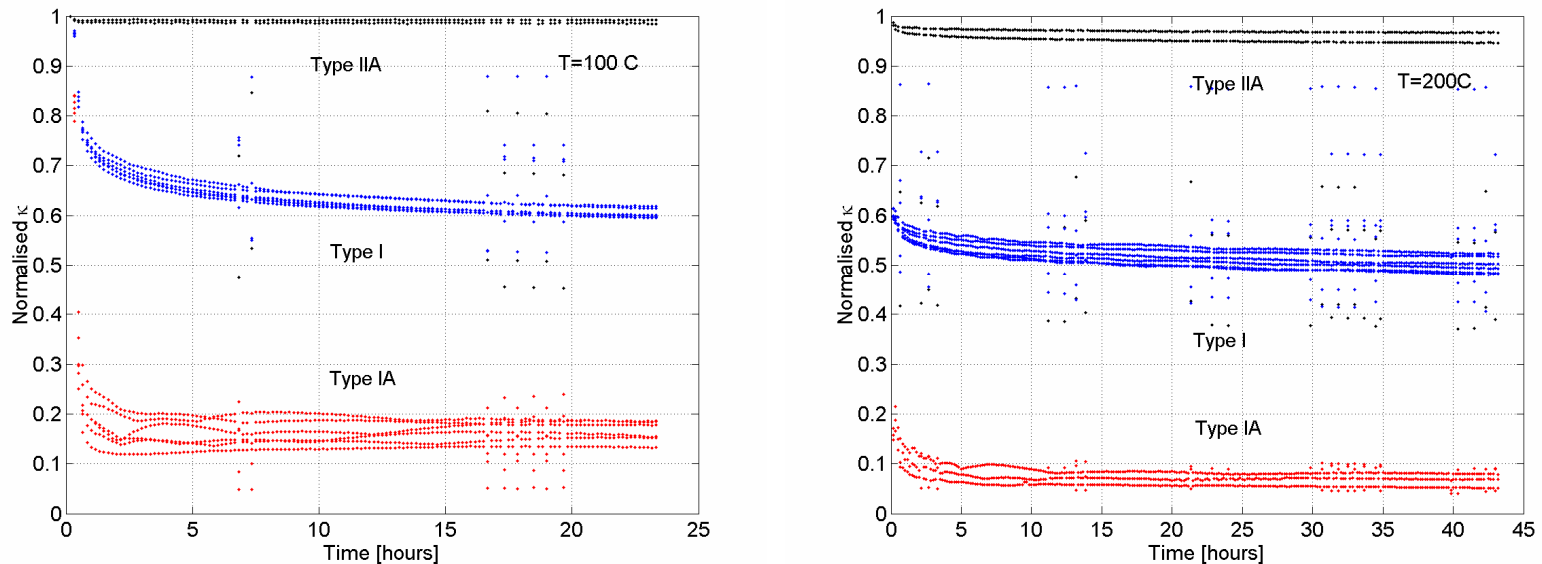

Figure. 9. The decay in the normalised integrated coupling constant by Type IIA (top), Type I (middle) and Type IA (bottom) gratings during heating at $100^{\circ} \mathrm{C}$ (left) and $200^{\circ} \mathrm{C}$ (right). This figure shows that the Type IA gratings present the lowest thermal stability, being erased at only modest temperatures. This has profound implications on the potential uses of IA gratings.

This data shows a vital result concerning the high temperature stability of Type IA gratings, indicating that IA gratings undergo a drastic initial decay at $100^{\circ} \mathrm{C}$ which implies that they are thermally unstable at this temperature. Figure 9 also confirms well known reports as to the stability of different types of Bragg grating showing that IIA gratings are more stable than Type I gratings. 


\section{EFFECT OF STRAIN ON TEMPERATURE STABILITY}

As an extension to our previous studies we inscribed Type IA Bragg gratings under strain, as it has been well documented that strain can affect the growth dynamics of Bragg gratings during manufacture ${ }^{17}$, and it was considered that strain would particularly impact the Type IA grating as it is characterised by a broad and shallow trap distribution. We used batches of highly hydrogenated Fibrecore PS1250/1500 fibre and recorded three grating Types into the same fibre section. In this way the fibre position relative to the phase mask and focussed inscription laser beam remained constant, thereby ensuring that the inscription procedure was repeatable. A Type IA grating was written without strain using the characteristic UV blank beam exposure method, as outlined earlier in this paper. A second (Type I) grating was written adjacent to the first, followed by a third Type IA grating inscribed under a constant strain of $1 \mathrm{~m} \varepsilon$ that was applied throughout the inscription procedure. This procedure was repeated for a second time and the fibres underwent thermal annealing for extended time periods. Relevant data is shown in Figure 10, confirming that the Type IA grating written under strain survived annealing to $180^{\circ} \mathrm{C}$, whereas the unstrained Type IA grating was completely erased at close to $100^{\circ} \mathrm{C}$. The figure shows data shifted along the wavelength axis to compensate for temperature induced wavelength shifts, to more clearly show the improved high temperature stability of the strained Type IA grating.

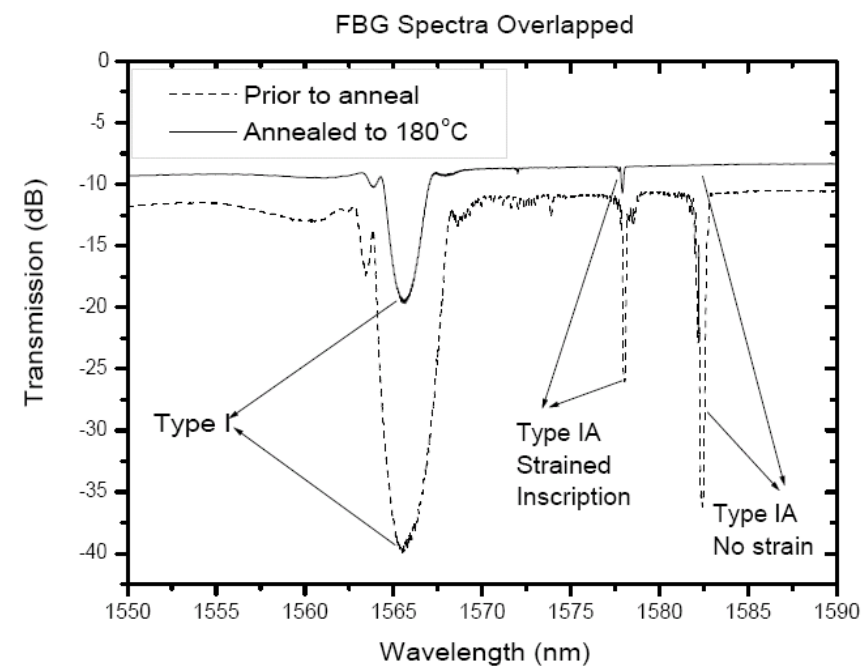

Figure 10. The transmission spectra of Type I, IA (inscribed without strain), IA (inscribed with strain) gratings prior to and after annealing to $180^{\circ} \mathrm{C}$. The data shows the improved temperature stability of the Type IA grating. Note the data is shifted along the wavelength and transmission axes to separate the curves and compensate for temperature induced wavelength shifts, for the sake of clarity. 


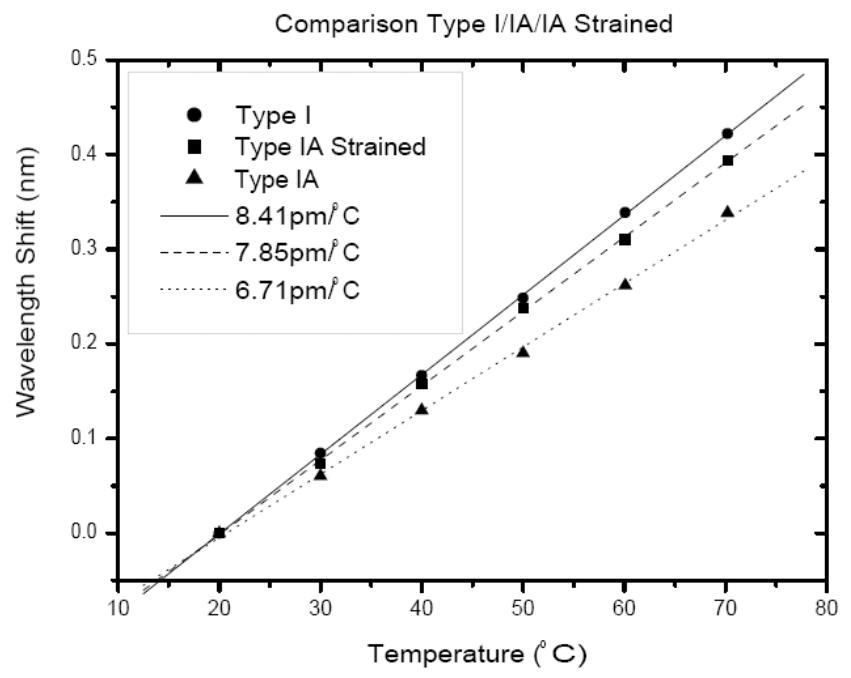

Figure 11. The difference in wavelength to temperature responsivity of Type I, IA, IA (inscribed with strain) gratings. It is observed that the strain increases the temperature coefficient of the Type IA grating and it behaves more like a Type I grating.

Furthermore, Figure 11 shows that straining the Type IA grating during inscription increases the wavelength to temperature responsivity, therefore there is the possibility of using strain to tailor the temperature coefficient of the Type IA grating for temperature compensated strain sensing applications. The effect of strain also hints at the role of defects on the grating stability with temperature and indicates an element of densification during inscription. This is under further investigation.

\section{CONCLUSIONS}

We have presented data that shows a fundamental result concerning the high temperature stability of Type IA gratings, which undergo a drastic initial decay at $100^{\circ} \mathrm{C}$, implying that they are thermally unstable at this temperature. However, the modulated index change of IA gratings remains stable at lower annealing temperatures of $80^{\circ} \mathrm{C}$, and the mean index change decays at a comparable rate to Type I gratings at $80^{\circ} \mathrm{C}$, this sets a maximum temperature for which IA gratings may be reliably used as less than $80^{\circ} \mathrm{C}$. Furthermore, we confirm the stability of different types of Bragg gratings showing that Type IIA gratings are more stable than Type I gratings. However, further studies show that straining the fibre throughout the inscription process leads to a robust Type IA grating surviving temperatures exceeding $180^{\circ} \mathrm{C}$. Therefore, the combination of Type I and IA (strained) gratings make it possible to decouple temperature and strain over larger temperature excursions.

\section{ACKNOWLEDGEMENTS}

The authors acknowledge the COST270 action "Reliability of optical components and devices in communications networks and systems" for support of this work through the funding of a Short Term Scientific Mission.

\section{REFERENCES}

[1] A.G. Simpson, K. Kalli, K. Zhou, L. Zhang and I. Bennion, "Formation of type IA fibre Bragg gratings in germanosilicate optical fibre," Electronics Letters, Vol. 40, p 163-164, 2004.

[2] A.G. Simpson, K. Kalli, L. Zhang, K. Zhou and I. Bennion, "Abnormal photosensitivity effects and the formation of type IA FBGs,” BGPP, Monterey, California, MD31, 2003.

[3] A.G. Simpson, K. Kalli, K. Zhou, L. Zhang and I. Bennion, "An idealised method for the fabrication of temperature invariant IA-I strain sensors," postdeadline session, OFS16 Nara, Japan, PD4, 2003.

[4] P. Lemaire, "Reliability of optical fibres exposed to hydrogen: prediction of long-term loss increases," Optical Engineering, Vol. 30, p 780-781, 1991. 
[5] D. L. Williams and R. P. Smith, "Accelerated lifetime tests on UV written intracore gratings in boron germania codoped silica fibre,” Electronics Letters, vol. 31, pp. 2120-2121, 1995.

[6] S. Kannan, J. Z. Y. Guo, and P. J. Lemaire, "Thermal stability analysis of UV-induced fibre Bragg gratings," Journal of Lightwave Technology, Vol. 15, pp. 1478-1483, 1997.

[7] S. Ishikawa, A. Inoue, M. Harumoto, T. Enomoto, and H. Kanamori, "Adequate aging condition for fibre Bragg grating based on simple power low model," presented at Optical Fibre Sensors (OFS1998), 1998.

[8] H. Patrick, S. L. Gilbert, A. Lidgard, and M. D. Gallagher, "Annealing of Bragg gratings in hydrogen-loaded opticalfibre," Journal of Applied Physics, Vol. 78, pp. 2940-2945, 1995.

[9] M. Aslund and J. Canning, "Annealing properties of gratings written into UV-presensitized hydrogen-outdiffused optical fibre," Optics Letters, Vol. 25, pp. 692-694, 2000.

[10] P. Niay, P. Bernage, S. Legoubin, M. Douay, W. X. Xie, J. F. Bayon, T. Georges, M. Monerie, and B. Poumellec, "Behavior of Spectral Transmissions of Bragg Gratings Written in Germania-Doped Fibres - Writing and Erasing Experiments Using Pulsed or Cw Uv Exposure,” Optics Communications, Vol. 113, pp. 176 -192, 1994.

[11] T. Erdogan, V Mirzahi, P. Lemaire, and D. Monroe, "Decay of ultraviolet-induced fibre Bragg gratings," Journal of Applied Physics, Vol. 76, pp. 73-80, 1994.

[12] S. R. Baker, H. N. Rourke, V. Baker, and D. Goodchild, "Thermal decay of fibre Bragg gratings written in boron and germanium codoped silica fibre ," Journal of Lightwave Technology, Vol. 15, pp. 1470-1477, 1997.

[13] Y. Masuda and et. al, "Wavelength evolution of fibre Bragg gratings fabricated from hydrogen loaded optical fibre during annealing," Journal of Lightwave Technology, Vol. 22, pp. 934-941, 2004.

[14] K. E. Chisholm, K. Sugden, and I. Bennion, "Effects of thermal annealing on Bragg fibre gratings in boron/germania co-doped fibre," Journal of Physics D-Applied Physics, Vol. 31, pp. 61-64, 1998.

[15] S. Pal, J. Mandal, T. Sun, and K. T. V. Grattan, "Analysis of thermal decay and prediction of operational lifetime for a type I boron-germanium codoped fibre Bragg grating,” Applied Optics, Vol. 42, pp. 2188-2197, 2003.

[16] J. Rathje, M. Kristensen and J. E. Pedersen, "Continuous anneal method for characterizing the thermal stability of ultraviolet Bragg gratings," Journal of Applied Physics Vol. 88, pp. 1050-1055, 2000.

[17] A. Othonos and K. Kalli "Fiber Bragg Gratings: Fundamentals and Applications in Telecommunications and Sensing", Artech House, Boston, London, 1999. Chapter 2, section 2.5.1.2.

*kkalli@cytanet.com.cy; phone 35722406537; fax 35722406545 